\title{
Achieving Effective Power System Observability in Optimal PMUs Placement Using GA-EHBSA
}

\author{
L. Parimalam*, R. Rajeswari \\ Department of EEE, Government College of Technology, Coimbatore, India \\ Email: "parimalamphd@gmail.com
}

Received 20 February 2016; accepted 15 March 2016; published 29 June 2016

Copyright @ 2016 by authors and Scientific Research Publishing Inc.

This work is licensed under the Creative Commons Attribution International License (CC BY). http://creativecommons.org/licenses/by/4.0/

c) (i) Open Access

\begin{abstract}
Normally, the power system observation is carried out for the optimal PMUs placement with minimum use of unit in the region of the Smart power grid system. By advanced tool, the process of protection and management of the power system is considered with the measurement of time-synchronized of the voltage and current. In order to have an efficient placement solution for the issue, a novel method is needed with the optimal approach. For complete power network observability of PMU optimal placement a new method is implemented. However, the process of placement and connection of the buses is considered at various places with the same cost of installation. GA based Enhanced Harmony and Binary Search Algorithm (GA-EHBSA) is proposed and utilized with the improvement to have least PMU placement and better optimization approach for finding the optimal location. To evaluate the optimal placement of PMUs the proposed approach is implemented in the standard test systems of IEEE 14-bus, IEEE 24-bus, IEEE 30-bus, IEEE 39-bus and IEEE 57-bus. The simulation results are evaluated and compared with existing algorithm to show the efficient process of optimal PMUs placement with better optimization, minimum cost and redundancy than the existing.
\end{abstract}

\section{Keywords}

IEEE Bus System, Searching Algorithm, GA, Harmony, Observability, Optimal PMU Placement

\section{Introduction}

Synchronized phasor measurement is a primary technology that can upgrade the monitoring and measurement of critical aspects of the Electric Power System, real time monitoring, control and system state estimation.

\footnotetext{
${ }^{*}$ Corresponding author.
} 
Synchronized measurements of real-time branch currents and bus voltages are provided by PMU. In 1980s PMUs were introduced and established it as an ultimate data acquisition technology, which is applied in wide area measurement systems with many applications that are currently under development. PMUs access synchronized measurements. These measurements are enabled using global positioning system (GPS) with better accuracy than one microsecond. By sampling the voltage and the current waveforms simultaneously, Synchronicity among PMUs is achieved.

At the same time a common synchronizing signal from the Global Positioning System (GPS) is used to attain synchronicity. To improve the performance of monitoring, control system in various fields phasors from different buses of a power system is obtained with the same time-space. Power system monitoring, operation and management require system state variables estimation; it is generally obtained by state estimation. The main responsibility of the state estimation is obtaining complete and reliable data. This is needed for multiple programs in controlling the power network. Already, supervisory control and data acquisition (SCADA) system and network of remote terminal units (RTUs) provided state estimators to process measurements which include power flows, injections and bus voltage magnitudes.

The main issue of the SCADA system is difficult in measuring from far distance. Real time power control network requires a real-time wide area monitoring, protection, and control (WAMPAC) system which utilize synchronized measurement technology (SMT). WAMPAC system is enabled by PMUs to contribute real-time measurements of bus voltage and branch current phasors. When the PMUs are installed on all buses, the power system can be completely examined, but this is non-economical because the PMU is a high cost instrument. To reduce the cost the PMU is installed in proper buses. So that the optimal number of PMU is needed to be accessed to obtain an entire observable power in the network desires an appropriate optimization technique which has been widely managed by a number of researchers in recent years [1].

An exact methodology is required to regulate the optimal placements of PMUs in a power system network. A protective action in a power system is included in PMU in addition to its ability to measure voltage and current phasor. The aim of the present paper is curtailed to find out the optimal location of PMUs in power system initially and then the usage of PMUs is estimated for state estimation.

A power system is determined as completely observable only when all the states are uniquely resolved. The intention of the present work is finding the minimum number of PMUs to make the system allegorically observable and also to determine the optimal locations of these PMUs. Still now, there is going on a great research to find the minimum number of PMUs and their optimal locations.

A bisecting search method is achieved to asset the minimum number of PMUs to generate the system completely observable. In bisecting search, to choose the placement set randomly simulated annealing method is employed. To obtain optimal PMU locations, the author uses a simulated annealing technique in their graphtheoretic procedure. In search process, there is a possibility for a placement set to make the system observable and can be overlooked. The optimal PMU locations can be estimated using genetic algorithm. The minimum number of PMUs can be determined by using integer programming. However, this method has a problem of being trapped in local minima. The main disadvantage is that, from the beginning, the method may conclude to have only one single solution, while there is a chance of existing more than one solution. Integer programming cannot handle multiple objectives, such as reducing the PMUs and increasing the measurement redundancy.

In this paper, a new approach is proposed to obtain optimal placement of minimum PMUs. To determine the optimal location of the PMUs heuristic search method is engaged. The eventual target lies in finding the minimum number of PMUs and their corresponding locations. The proposed algorithms are developed in MATLAB. The rest of the paper organised in a section wise. In section the related work survey is carried out with the techniques for optimal placement of PMUs. In Section 3, the proposed technique is explained with the implementation and the performance analysis of the proposed approach is presented in Section 4. Finally, the conclusion of the proposed searching approach is presented in Section 5.

\section{Related Work}

In this section, the literature survey of the optimal PMUs placement and related searching algorithms are discussed. In recent power system the process of control, protection and the operation is considered and provides the real time phasor of current branch and the bus voltage synchronized measurements [1].

Real time Monitor provides reliable and secure power system operation. By Predictable Supervisory Control and Data Acquisition/Energy Management System (SCADA/EMS), the data are illustrated with the time interval 
of 2 - 10 sec. It is obtained by Predictable Supervisory Control and Data Acquisition/Energy Management System (SCADA/EMS). Using PMU, the Synchronized Phasor Measurement (SPM) based Wide Area Monitoring System (WAMS) illuminates in the network at various locations. The Global Positioning System (GPS) satellites are recycled to coordinate them and develop the strategy for efficient optimal location and placement [2].

Generally, Phasor measurement units (PMUs) are reflected as a controlling tool and used to optimize the locations of the PMU. It is observed and resolved the issues by binary integer linear programming (BILP). The measurement of flow and injected power impacts is accommodated by this approach and also it removes the loss of PMU in the allocation of optimal PMU by the decision strategy. The methodology of Integer Programming is used to have observability and minimum cost for installation. Also, it is processed instead of Phasor Measurement Unit (PMU). In zero injection, the PMUs are minimized and it is not properly handled the neighboring buses [3] [4].

The Genetic Algorithm (GA) based approach makes reliable placement in smart grid with the combine of two inconsistent objectives. It provides maximum reliability and minimum placement of PMU. It is employed for large scale system bus issues and it develops the issues of multi-objective as a nonlinear optimization problem [5]. The boost DC-DC convertor topology having the exclusive ability of terminating the ripple input during the random reselection of duty cycle [6]. The linear algorithm is employed for complete observability of system by identifying the least number of phasor measurement units (PMUs) [7]. The complete system synchronized snap shot required for the diagnosis of fault and state emission. For OPP problematic, the nonlinear programming and a sequential quadratic programming method are formulated using binary integer programming (BIP) model. Based on NLP the feasibility is investigated for obtaining optimal solution. The System Observability Redundancy Index is measured for ranking the multiple solutions [8].

In the network of power system the PMU deployment takes place with the dynamic process of operating conditions for monitoring. The estimated issue is converted from non- linear iterative state to linear non iterative state. In a huge power network the installation of PMU is a difficult task to schedule in a single stage [9]. To overcome this problem the installation is carried out in multiple stages because it has infinite capacity of channels. Phasor Measurement Units (PMUs) are the major essentials in confident process of managing and observation of the power network. The observability of the power grid system for placing PMU with minimum cost is required. It covers a maximum area of network with limited resources, but it doesn't reach the full observability. The problem codified as an integer linear programming (ILP) model and it is solved for optimality [10].

The leading difficult problematic is the process of system logic operation and design. The logical system is optimized by the economic factors. The heuristic techniques using the network logic system are developed and knapsack problem is associated with enormous number of logistic issues. The harmony search based algorithm is involved to solve the issues and it is a programming language of fourth-generation. Also, it improved the bandwidth correction method with the possible control or convergence modification of the algorithm [11].

The wide area measurement system (WAMS) is based on synchronized PMUs for a hybrid smart AC/DC power networks in a grid-connected PV-power plants. The main objective is to increase the system reliability by the major dependency on WAMS. It acts as a distributed intelligence agent in the network of power with the capability improvement of protection, monitoring and control. In real time the bus data measurement is easily managed and validated in the applications smart grid [12].

In the placement of PMU various cases are represented and zero injection buses are carried out to reduce the needed of PMU. By single failure of PMU makes the reliability of the system with improved. In order to resolve the failure instead of single PMU each bus is considered with two PMUs at least. By using WLS and LAV, the system estimation and the bus system phase angles and voltages are related the PMU with and without. After estimation, it is clear that the LAV is well than WLS and it minimize the errors when including PMU. For the analysis of voltage stability the data are used and processed by using two indices-FVSI and LQP. The possibility study for random selection of lines with a stressful situation is carried out under various condition of operating for obtaining ideas [13].

The graph theory and mathematical programming are processed to overcome the issues and also Heuristic method is implemented with the approach. The ideal placement of Phasor Measurement Unit of the system is considered to have full observation and less PMUs. The location finding is processed by using Heuristic Search method. It is used to resolve the issues of connectivity of system network and heuristics process [14].

\section{Proposed Work}

In this section, GA based Enhanced Harmony and Binary Search Algorithm (GA-EHBSA) is proposed and 
implemented to have a least PMU placement and optimal location by using enhanced optimization approach. The observability of the power system is processed by numerical and topological wise. To employ topological observability the numerical process is carried out from the estimation of matrix.

The topological process is utilized for the optimal solution by using the rules. The instructions consist between buses; first, the direct measurement of voltage and current by ensuring buses with the installation of PMU. Second, estimation of phasor voltage and current is carried out at different end of the line and the third rule is the measurement of voltage and current is estimated at both ends of the line via pseudo or direct measurement. The incident branches of current and the voltage is measured by the preparation of PMU in the buses. As per the rules, the buses are interconnected with each other and considered the nearest branch details in matrix form to know the connectivity of the buses. In the information of topology power system, the system observability is utilized and formatted in a connectivity matrix $(M)$.

$$
M(i, j)= \begin{cases}1, & \text { if } i \text { is equal to } j \\ 1, & \text { if } i \& j \text { connected } \\ 0, & \text { otherwise }\end{cases}
$$

The binary state vector elements are presented as below and formulated the objective functions of placement for optimal PMU by applying proposed approach.

$$
X(i)= \begin{cases}1, & \text { if the equipedbus of PMU is } i \\ 0, & \text { otherwise }\end{cases}
$$

The searching process of optimal location is processed by using Enhanced Harmony and Binary Search Algorithm based on GA. Here, GA is a technique of generating the vector within the limit of interval to obtain global solution. The initial process is GA is continuing after the function of generating power and the estimation of transmission loss. The vector element generates the outcomes of real power as per the limitation of minimum and maximum range. The relative fitness function is obtained by evaluating the cost value of fitness.

The best vector is chosen based on the cost function and the value is minimum when compare to other values. The minimum cost vector is taken as parent for power generating. The function of crossover and mutation is carried out by the non-uniform arithmetic process. Only least value of mutation outcomes and it is processed randomly to form chromosomes. The best solution is obtained in the generation by searching process. It will repeat the process of generation until it reaches the maximum process and criteria.

In the proposed approach, the searching algorithm is based on the function of genetic algorithm. The searching approach is the Harmony and Binary Search Algorithm. It is a meta-heuristic process to solve the issues of optimization. In order to obtain better harmony the proposed approach is implemented and also solution for economic load dispatch. The objective function of the optimization is represented by the upper and lower bound of decision variable. It is evaluated based on the function given in Equations ((4) and (5)) respectively.

Minimization $f(x)$

Subject to $x_{i} \in X_{i}, \quad i=1,2, \cdots, N$

$$
\begin{gathered}
L X_{i}<X_{i}<U X_{i} \\
\gamma p(T) \geq \frac{k+2}{3} \\
\gamma p(T) \leq \frac{n}{3}
\end{gathered}
$$

The location variables of the decision set $(H)$ are initialized randomly with the generated vectors.

$$
H=\left[\begin{array}{ccccc}
X_{1,1} & X_{1,2} & \cdots & X_{1, n-1} & X_{n} \\
X_{2,1} & X_{2,2} & \cdots & X_{2, n-1} & X_{2, n} \\
\vdots & \vdots & \ddots & \vdots & \vdots \\
X_{s-1,1} & X_{s-1,1} & \cdots & X_{s-1, n-1} & X_{s-1, n} \\
X_{s, 1} & X_{s, 1} & \cdots & X_{s, n-1} & X_{s, n}
\end{array}\right]
$$


According to the fitness function the worst and best case of harmony is defined and the worst case is replaced and ignored form the function. As per the limitation process the function is repeated and it will terminate once it reaches the optimal solution else it begin from the probability function. For utilization of proposed searching process is carried out with the binary strategy. The strategies are included in the parameters of harmony for the random selection of vectors. As per bit selection strategy, the vector is decided form the harmony in a random process when the elements selected. Here, it consists for each bit of the Harmony.

$$
\begin{gathered}
N H_{b j}= \begin{cases}x_{b j} & \text { if rand } 1<\text { Memory rate } \\
R & \text { else }\end{cases} \\
R= \begin{cases}0 & \text { if rand } 2<0.5 \\
1 & \text { else }\end{cases}
\end{gathered}
$$

According to individual strategy of selecting the process is carried out within the group of harmony and the random function is same. Here, the random integer is considered between 0 and 1 and the selection process between 1 to size.

$$
\begin{gathered}
N H_{h j}= \begin{cases}x_{h j} & \text { if rand } 1<\text { Memory rate } \\
R & \text { else }\end{cases} \\
R= \begin{cases}0 & \text { if rand } 2<0.5 \\
1 & \text { else }\end{cases}
\end{gathered}
$$

$b \& h \in\{$ harmony memory size 1 to $s\}$

In the proposed algorithm, the functions are changed with the condition for the improvement of procedure process and efficient access. Here, the creation is performed randomly as per the function given below.

$$
\begin{gathered}
N H_{h j}= \begin{cases}x_{h j} & \text { if rand } 1<\text { Memory rate } \\
R & \text { else }\end{cases} \\
R= \begin{cases}0 & \text { if rand } 2<0.5 \\
\operatorname{randi}[0,1] & \text { else }\end{cases}
\end{gathered}
$$

The proposed approach procedure is given below:

1) Begin with the placement of PMU in bus and estimate the limitation of upper and lower bound.

2) Read the Data and solve the OPF using GA

3) Population - initialization

4) Evaluate the beginning stage

5) Check the condition of maximum generation. If condition satisfied proceed with the searching approach.

6) Else if not satisfied then select the strategy of individuals

7) Evaluate the non-uniform architecture function of crossover and mutations and applied to the population. As per the mutation function the estimation is carried out.

8) Evaluate the new individuals and continue with the maximum generation condition checking.

9) The outcomes is obtained and updated in memory.

10) The GA outcome is considered as a starting point to solve OPF issues by applying HBSA approach.

11) Create harmony initial memory

12) Create harmony vector by considering random selection with the memory consideration and adjustment of pitch.

13) Get results and Update the harmony memory

14) Check the criteria of termination, if not satisfied go to step 10.

15) Else move to system observation checking as per the condition.

16) Obtain the optimal solution and evaluate the extreme redundancy index (ISORI).

17) Attain absolute solution.

The unnecessary process is avoided in the optimal solution in order to have a reduction of execution time. In 


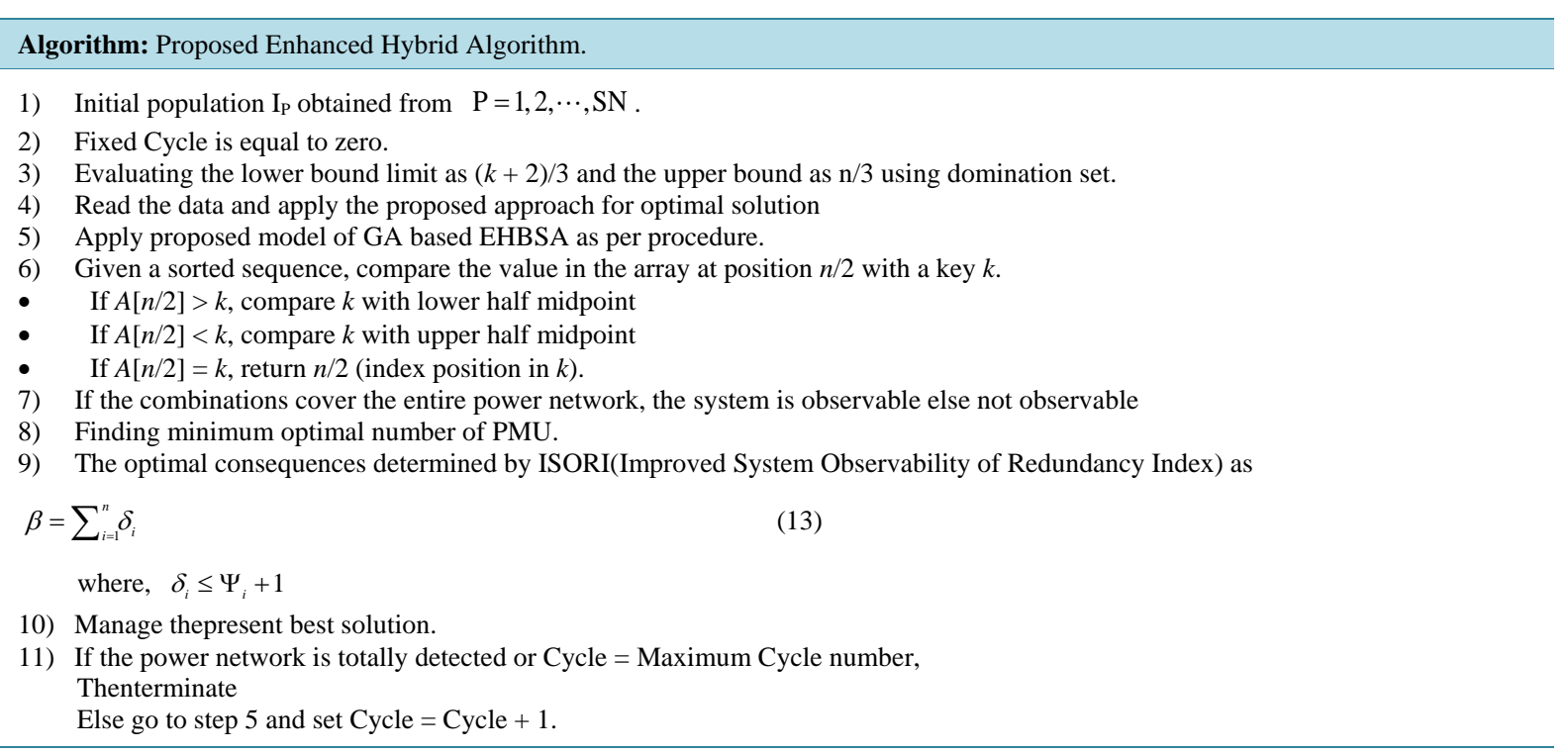

the proposed algorithm the Improved System Observability Redundancy Index is functional for substantial selection procedure of efficient solution and to have efficient system monitoring. It is performed with the IEEE standard bus system bound function. In the connectivity matrix A, the bus interconnectivity is presented.

\section{Simulation Results}

In this section, the simulation results of the GA based Enhanced Harmony and Binary Search Algorithm (GA-EHBSA) is obtained and analyzed the performances to show better efficiency than the existing. By using proposed GA-EHBSA, the significant development of PMUs in the smart grid system is achieved and achieved the results by simulating the proposed algorithm in MATLAB/SIMULINK platform in the IEEE bus system. The location finding of PMUs, minimum execution time, better reliability and less cost are obtained from the simulation outcomes.

The connectivity matrix of A for the IEEE 14 bus system is given below. Figure 1 shows the comparison of various algorithms between cost and bus system. Table 1 shows the placement cost and the optimal redundancy index of the proposed system and existing systems. Better optimal solution with efficient reliable process is obtained by the proposed approach. The optimal placement of PMU for the IEEE bus system is shown in Table 2. Table 3 illustrates the outcomes of minimum PMU location finding performances for various algorithms.

$$
A=\left[\begin{array}{llllllllllllll}
1 & 1 & 0 & 0 & 1 & 0 & 0 & 0 & 0 & 0 & 0 & 0 & 0 & 0 \\
1 & 1 & 1 & 1 & 1 & 0 & 0 & 0 & 0 & 0 & 0 & 0 & 0 & 0 \\
0 & 1 & 1 & 1 & 0 & 0 & 0 & 0 & 0 & 0 & 0 & 0 & 0 & 0 \\
0 & 1 & 1 & 1 & 1 & 0 & 1 & 0 & 1 & 0 & 0 & 0 & 0 & 0 \\
1 & 1 & 0 & 1 & 1 & 1 & 0 & 0 & 0 & 0 & 0 & 0 & 0 & 0 \\
0 & 0 & 0 & 0 & 1 & 1 & 0 & 0 & 0 & 0 & 1 & 1 & 1 & 0 \\
0 & 0 & 0 & 1 & 0 & 0 & 1 & 1 & 1 & 0 & 0 & 0 & 0 & 0 \\
0 & 0 & 0 & 0 & 0 & 0 & 1 & 1 & 0 & 0 & 0 & 0 & 0 & 0 \\
0 & 0 & 0 & 1 & 0 & 0 & 1 & 0 & 1 & 1 & 0 & 0 & 0 & 1 \\
0 & 0 & 0 & 0 & 0 & 0 & 0 & 0 & 1 & 1 & 1 & 0 & 0 & 0 \\
0 & 0 & 0 & 0 & 0 & 1 & 0 & 0 & 0 & 1 & 1 & 0 & 0 & 0 \\
0 & 0 & 0 & 0 & 0 & 1 & 0 & 0 & 0 & 0 & 0 & 1 & 1 & 0 \\
0 & 0 & 0 & 0 & 0 & 1 & 0 & 0 & 0 & 0 & 0 & 1 & 1 & 1 \\
0 & 0 & 0 & 0 & 0 & 0 & 0 & 0 & 1 & 0 & 0 & 0 & 1 & 1
\end{array}\right]
$$




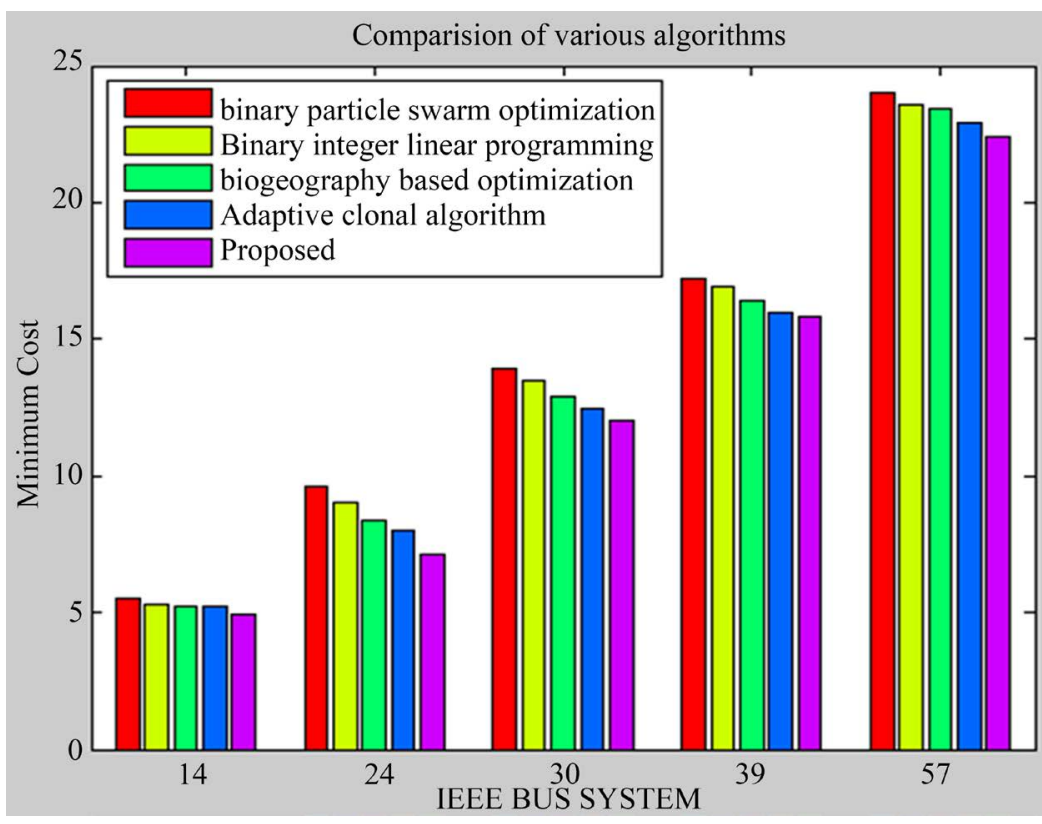

Figure 1. Cost vs bus system for various algorithms.

Table 1. Optimal PMU placement cost and redundancy for the IEEE test systems.

\begin{tabular}{ccc}
\hline Bus system & Cost & Redundancy \\
\hline IEEE 14-bus & 4.9 & 24 \\
IEEE 24-bus & 7.1 & 27 \\
IEEE 30-bus & 12 & 58 \\
IEEE 39-bus & 15.8 & 66 \\
IEEE 57-bus & 22.4 & 71 \\
\hline
\end{tabular}

Table 2. Optimal PMU placement for the IEEE test systems.

\begin{tabular}{ccc}
\hline Bus system & PMUs location & Number of PMUs \\
\hline IEEE 14-bus & $2,7,10,13$ & 4 \\
IEEE 24-bus & $2,3,8,10,16,21,23$ & 7 \\
IEEE 30-bus & $1,6,7,10,11,12,18,26,29$ & 9 \\
IEEE 39-bus & $2,6,9,12,14,17,22,23,29,32,33$ 34, 37 & 13 \\
IEEE 57-bus & $1,4,9,15,20,24,25,28,29,32,36,38,41,46,50,53,57$ & 17 \\
\hline
\end{tabular}

Table 3. Comparisons of various algorithms for IEEE bus system cost.

\begin{tabular}{|c|c|c|c|c|c|}
\hline Algorithm & $14^{\text {th }}-$ Bus & $24^{\text {th }}-$ Bus & $30^{\text {th }}$-Bus & $39^{\text {th }}$-Bus & $57^{\text {th }}-$ Bus \\
\hline binary particle swarm optimization & 5.5 & 9.6 & 13.9 & 17.2 & 24 \\
\hline Binary integer linear programming & 5.3 & 9 & 13.5 & 16.9 & 23.6 \\
\hline biogeography based optimization & 5.2 & 8.4 & 12.9 & 16.4 & 23.4 \\
\hline Adaptive clonal algorithm & 5.2 & 8 & 12.5 & 16 & 22.9 \\
\hline Proposed & 4.9 & 7.1 & 12 & 15.8 & 22.4 \\
\hline
\end{tabular}

The simulation analysis of the weight array for the standard IEEE bus system of 14, 24, 30,39 and 57 is shown in Figures 2-6 respectively. As well as, the performances of the time requirement for the iteration in the standard IEEE bus systems (14, 24, 30, 39 and 57) are shown in Figures 7-11 correspondingly. 




Figure 2. Analysis of weight array in IEEE 14 bus system.

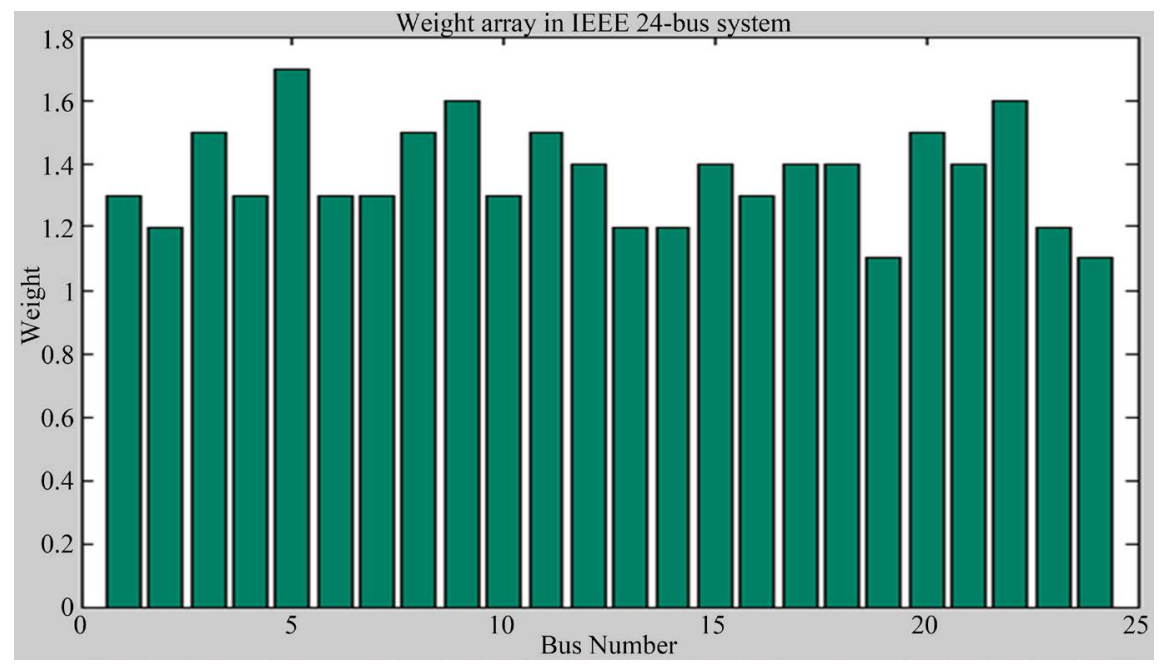

Figure 3. Analysis of weight array in IEEE 24 bus system.

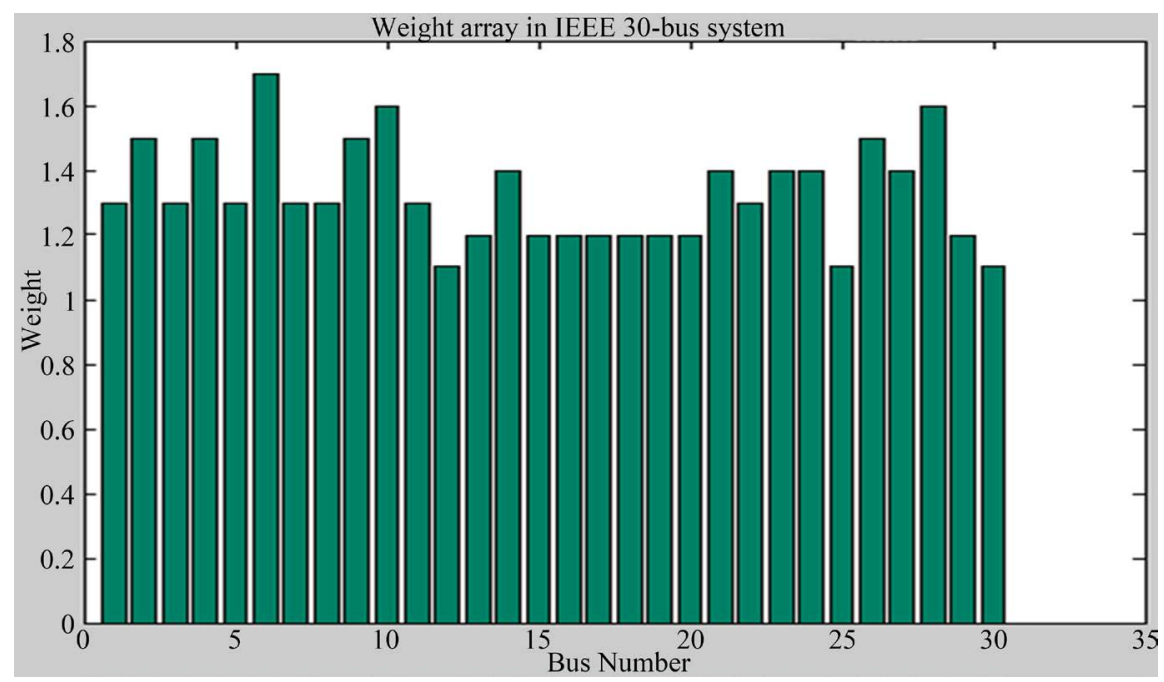

Figure 4. Analysis of weight array in IEEE 30 bus system. 


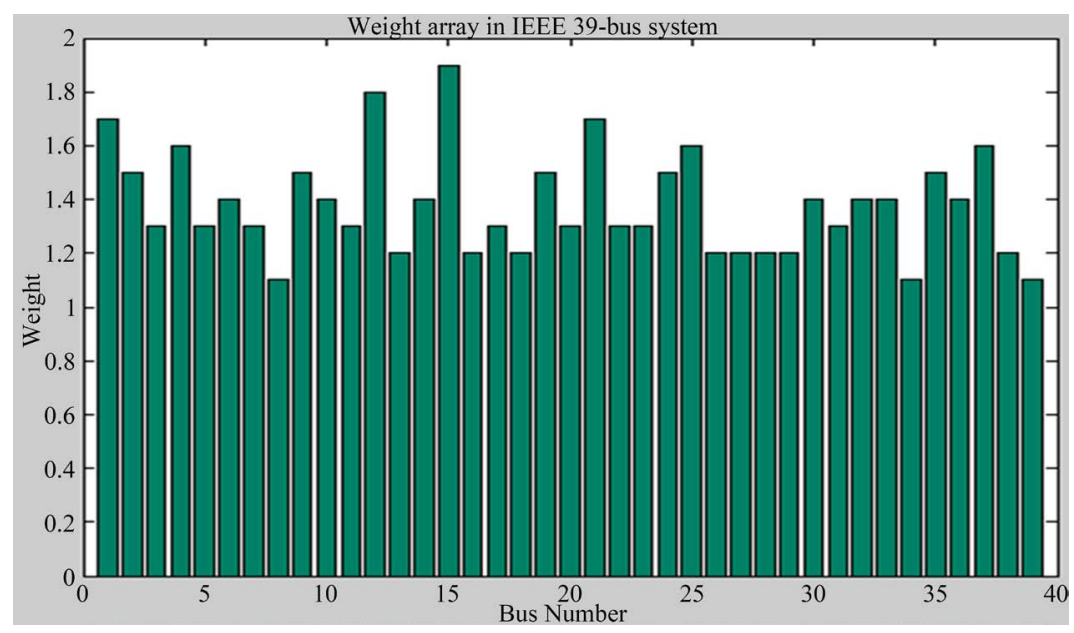

Figure 5. Analysis of weight array in IEEE 39 bus system.

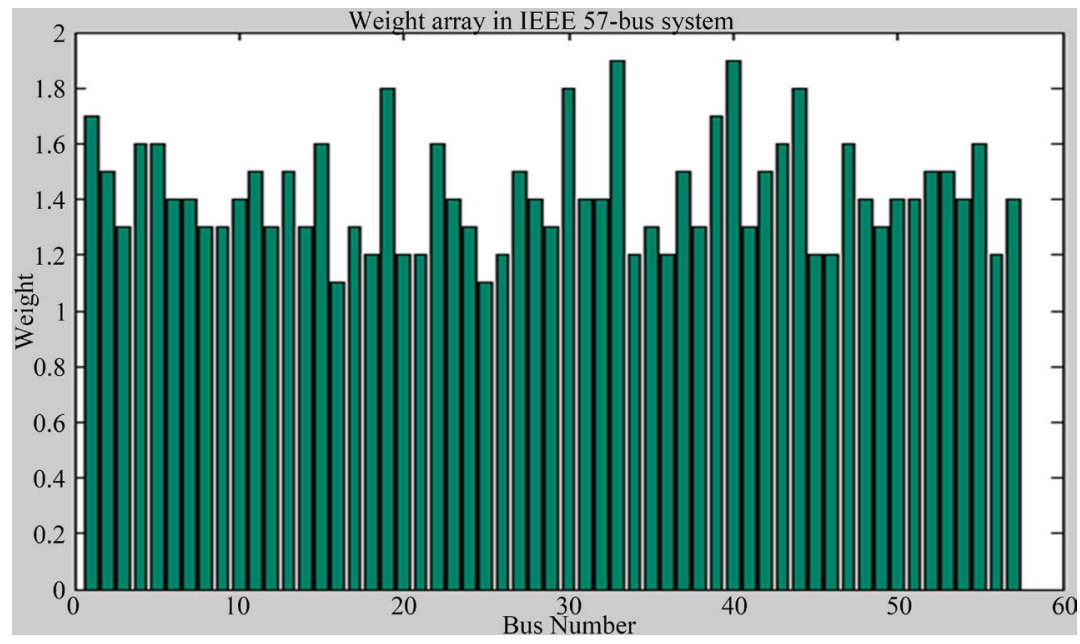

Figure 6. Analysis of weight array in IEEE 57 bus system.
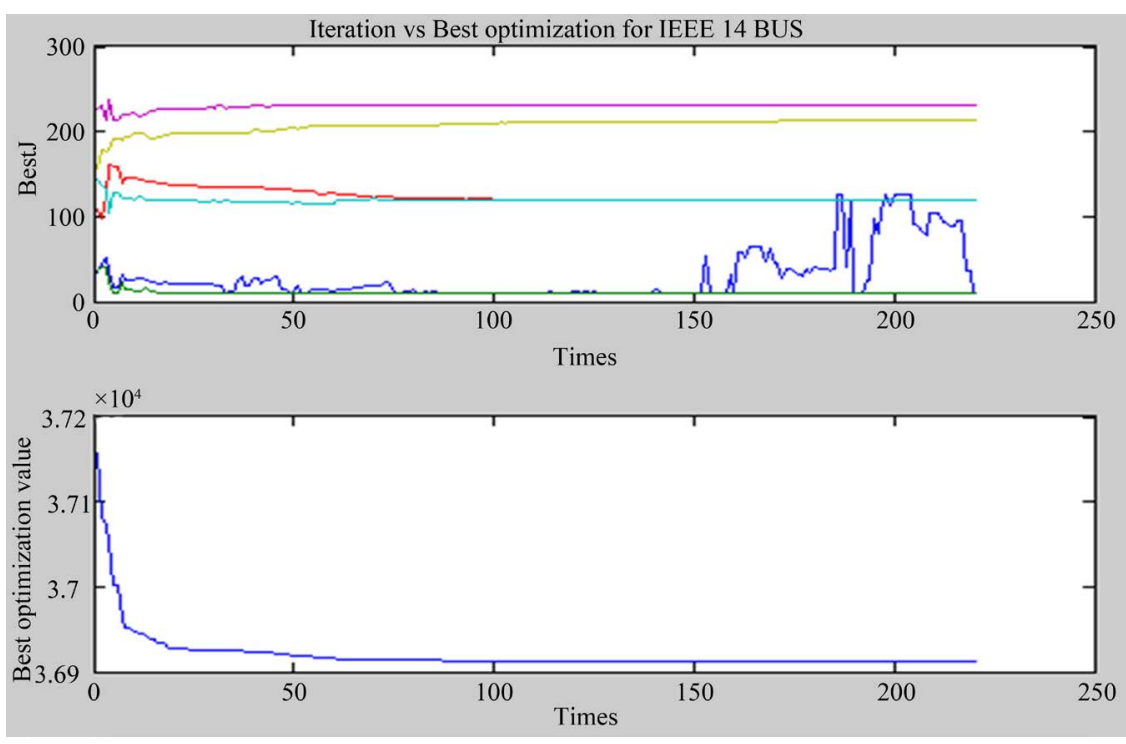

Figure 7. Simulation results of time for IEEE 14 bus system. 

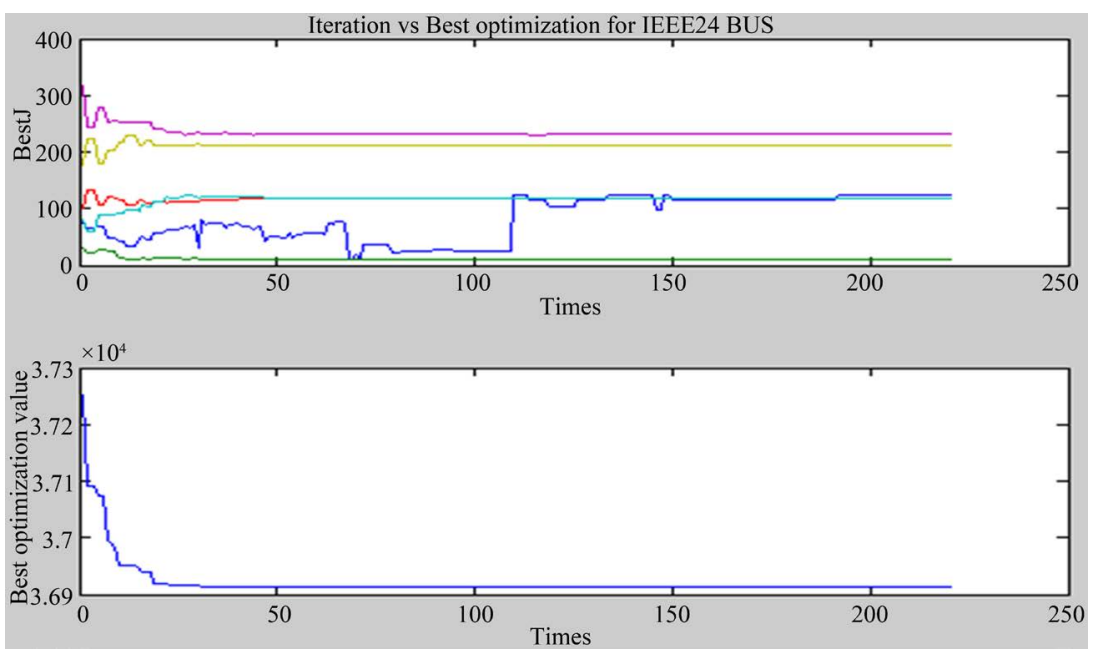

Figure 8. Simulation results of time for IEEE 24 bus system.
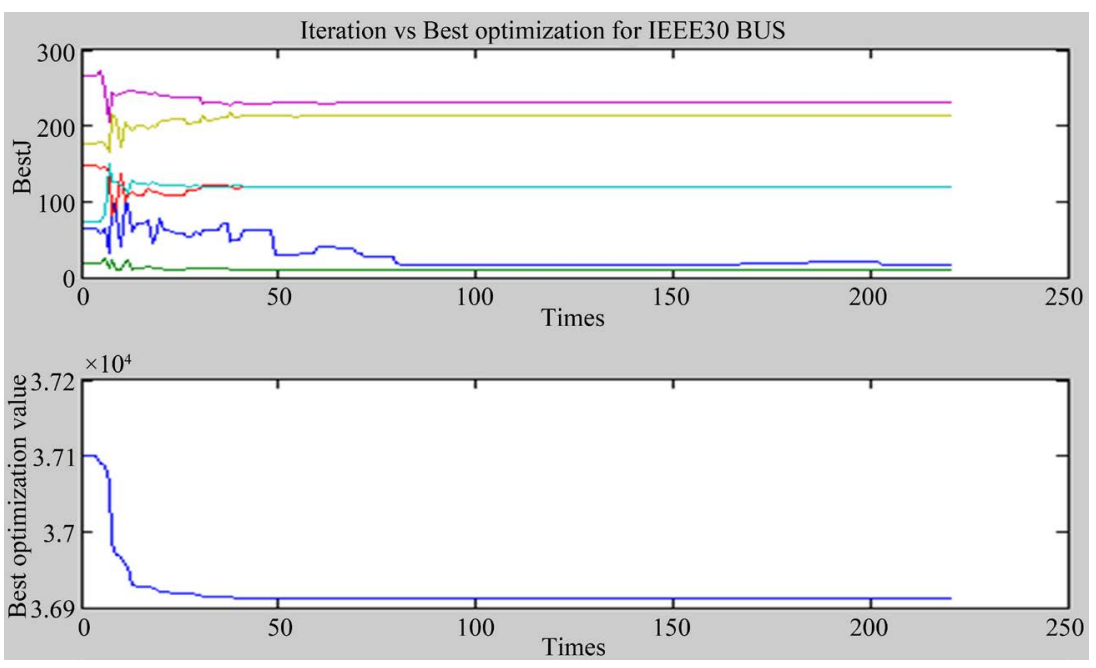

Figure 9. Simulation results of time for IEEE 30 bus system.
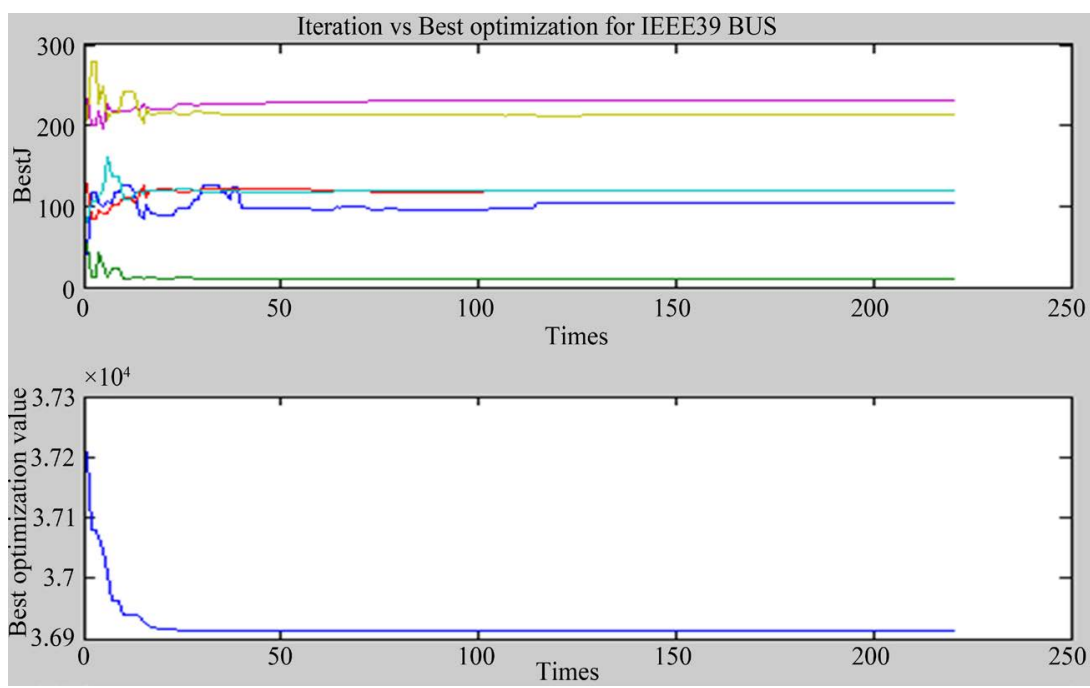

Figure 10. Simulation results of time for IEEE 39 bus system. 




Figure 11. Simulation results of Time for IEEE 57 bus system.

\section{Conclusion}

In this paper, GA based Enhanced Harmony and Binary Search Algorithm (GA-EHBSA) is proposed for efficient optimal placement of PMU and searching process. This paper introduces the approach as an optimization tool of meta-heuristic process for the placement issue solution. By this the system observability is carried out efficiently and has reliable process. The process of PMUs installation cost for various buses is illustrated based on the connected channels to the bus. The proposed approach was applied to IEEE 14, 24, 30, 39 and 57 bus standard test systems. The simulated outcomes conclude the efficiency of the proposed approach in finding location, less cost for PMUs installations and efficient access of complete observable of the power system.

\section{References}

[1] Nazari-Heris, M. and Mohammadi-Ivatloo, B. (2015) Application of Heuristic Algorithms to Optimal PMU Placement in Electric Power Systems: An Updated Review. Renewable and Sustainable Energy Reviews, 50, 214-228. http://dx.doi.org/10.1016/j.rser.2015.04.152

[2] Lashkari, H.D. and Sarvaiya, J.B. (2014) Matlab Based Simulink Model of Phasor Measurement Unit and Optimal Placement Strategy for PMU Placement. IJSRD_International Journal for Scientific Research \& Development, 2, 135-138.

[3] Singh, S.P. and Singh, S.P. (2014) Optimal PMU Placement in Power System Considering the Measurement Redundancy. Advance in Electronic and Electric Engineering, 4, 593-598.

[4] Abbasy, N.H. and Ismail, H.M. (2009) A Unified Approach for the Optimal PMU Location for Power System State Estimation. IEEE Transactions on Power Systems, 24, 806-813. http://dx.doi.org/10.1109/tpwrs.2009.2016596

[5] Khiabani, V., Erdem, E., Farahmand, K. and Nygard, K. (2014) Smart Grid PMU Allocation Using Genetic Algorithm. Journal of Network and Innovative Computing, 2, 30-40.

[6] Sathyasaraj, K. and Soundarajan, A. (2014) PV Fed Induction Motor Drive System Using Transformer Less High Gain Boost Converter. International Journal of Advanced Information Science and Technology (IJAIST), 26.

[7] Saini, R., Mam, M. and Saini, M.Kr. (2008) Optimal Placement of Phasor Measurement Units for Power System Observability. International Journal of Power System Operation and Energy Management, 2, 10-13.

[8] Theodorakatos, N.P., Manousakis, N.M. and Korres, G.N. (2014) Optimal PMU Placement Using Nonlinear Programming. OPT-i-An International Conference on Engineering and Applied Sciences Optimization, Kos Island, Greece, 4-6 June 2014.

[9] Rihan, M., Ahmad, M. and Beg, M.S. (2013) Optimal Multistage Placement of PMUs with Limited Channel Capacity for a Smart Grid. Electrical and Electronic Engineering, 3, 133-138. 
[10] Khiabani, V. and Farahmand, K. (2013) Max Covering Phasor Measurement Units Placement for Partial Power System Observability. Engineering Management Research, 2.

[11] Bányai, T. and Veres, P. (2013) Optimisation of Knapsack Problem with Matlab, Based on Harmony Search Algorithm. Advanced Logistic Systems, 7, 13-20.

[12] Amin, M.M., Moussa, H.B. and Mohammed, O.A. (2012) Wide Area Measurement System for Smart Grid Applications Involving Hybrid Energy Sources. Energy System, 3, 3-21.

[13] Sudha, R., Jain, D., Lahoty, U., Khushalani, S., Nivedita, G. and Jayabarathi, T. (2012) State Estimation and Voltage Stability Monitoring Using ILP PMU Placement. International Journal of Soft Computing and Engineering (IJSCE), 2.

[14] Shewale, R.H., Kethineni, B.K., Balaraju, U.P., Bhil, S.K. and More, P.D. (2012) Optimal Placement of Phasor Measurement Unit for Power System Observability by Heuristic Search Method. International Journal of Advanced Technology \& Engineering Research (IJATER), 2.

\section{Submit or recommend next manuscript to SCIRP and we will provide best service for you:}

Accepting pre-submission inquiries through Email, Facebook, Linkedin, Twitter, etc A wide selection of journals (inclusive of 9 subjects, more than 200 journals)

Providing a 24-hour high-quality service

User-friendly online submission system

Fair and swift peer-review system

Efficient typesetting and proofreading procedure

Display of the result of downloads and visits, as well as the number of cited articles

Maximum dissemination of your research work

Submit your manuscript at: http://papersubmission.scirp.org/ 